\title{
Initial Conditions Effect on Thermosolutal Natural Convection in a Horizontal Annular Porous Cavity
}

\author{
Ahmed Ja, Abdelkhalek Cheddadi \\ Mohammadia School of Engineers \\ Mohammed V University in Rabat, P. O. Box 765, Agdal, Rabat, Morocco \\ Jaahmed@hotmail.fr; cheddadi@emi.ac.ma
}

\begin{abstract}
The combined effect of different initial conditions types and variation of Rayleigh number on thermosolutal convection characteristics is numerically investigated in a horizontal annulus filled with a porous medium saturated by a binary fluid. The control parameters, radius ratio, $R=1.2$, Lewis number $L e=2$ and cooperating buoyancy forces, $N=2$, are considered in this investigation. Darcy's equation, conservation of the energy and species concentration equations are solved with the Alternating Direction Implicit (ADI) scheme, based on the Centered Finite Difference discretization. The steady-state numerical results using three types of initial conditions show that different multicellular flow patterns are obtained with increasing the Rayleigh number. The average Nusselt and Sherwood numbers behaviors are also discussed.
\end{abstract}

Keywords: Thermosolutal Convection, Porous Medium, Annular Space, Initial Conditions, Multicellular Flows, Heat and Mass Transfer Rates.

\section{Introduction}

As early as 1976s, natural convection in horizontal porous annulus heated from the inner cylinder and cooled from the outer one, has been numerically and experimentally investigated [1]. According to three initial conditions modes, the Galerkin method has been used to analyze the different bifurcations phenomena of thermal convection [2]. The resulting convergent solutions have proven the existence of a critical Rayleigh-Darcy number value from which the average Nusselt number has the same evolution whatever the initial conditions types used. In studies [3-4] a second order Finite Difference scheme has been employed, based on the Alternating Direction Implicit method and coupled with successive under relaxation to solve the two-dimensional Darcy-Boussinesq equations. For a radius ratio above 1.7 and Rayleigh numbers above a critical value, authors exhibit a closed hysteresis loop, indicating the presence of dual solutions from two to four convective cells, depending on the initial conditions used. The increase of Rayleigh number in the system of small radius ratio (i.e., below 1.7) involves a multicellular flow without the hysteresis loop behavior. The numerical results obtained in [5] predict the bifurcation point from unicellular flow to multicellular flow for the cavities of radii ratios $R=\sqrt{2}$ and 2 . Recently, the first- and second-order perturbed velocity and temperature fields are determined analytically in terms of Bessel functions based on heat conduction state profile [6]. The analysis shows that a transition of two convective cells to four cells is possible, then, the critical Rayleigh-Dacry number values for different thicknesses of the annular space are determined with accuracy. The numerical experiment of the Darcy-Brinkman model has been investigated in [7]. This work evaluated same bifurcation points for various Darcy number values.

Hence, the main objective of the present investigation is to evaluate the initial conditions effect on the flow structure and heat and mass transfer rates in a horizontal porous annulus. The annular space is filled with a porous medium saturated with a binary fluid. The inner and outer cylinders of radius, respectively, $r_{i}$ and $r_{o}$ are maintained at constant and uniform temperatures $\left(T_{i}, T_{o}\right)$ and concentrations $\left(S_{i}, S_{o}\right)$, with $T_{i}>T_{o}$ and $S_{i}>S_{o}$, as shown in Figure 1. The porous medium is considered to be homogeneous and isotropic and in local thermal and solutal equilibrium with the fluid. The flow field is assumed to be two-dimensional and laminar, also the binary fluid is considered to be incompressible and satisfying the Boussinesq approximation: $\rho=\rho_{0}\left[1-\beta_{T}\left(T-T_{0}\right)-\beta_{S}\left(S-S_{0}\right)\right]$, where $\rho_{0}$ is the reference density and $\beta_{T}$ and $\beta_{S}$ are the thermal and solutal expansion coefficients, respectively. All the results presented in this paper are for the radius ratio $R=$ 1.2, Lewis number $L e=2$ and buoyancy ratio $N=2$. 


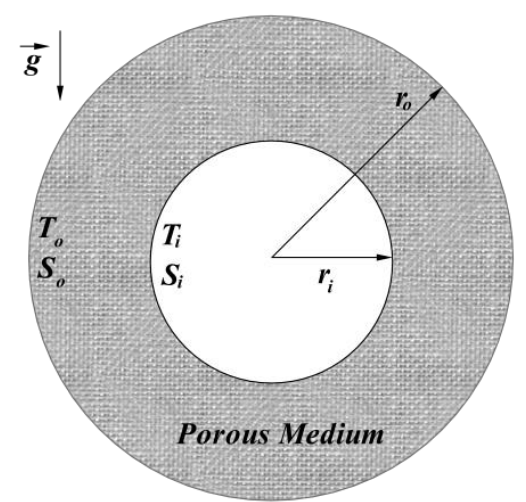

Fig. 1: Schematic diagram of the problem.

\section{Mathematic Formulation}

The two-dimensional governing equations that describe the laminar steady-state are Darcy's equation, conservation of energy and species concentration equations, which may be written in dimensionless form using stream function, $\Psi$, and the formulation is as follows:

$$
\begin{gathered}
\nabla^{2} \Psi=-R a\left[\left(\sin \varphi \frac{\partial T}{\partial r}+\frac{\cos \varphi}{r} \frac{\partial T}{\partial \varphi}\right)+N\left(\sin \varphi \frac{\partial S}{\partial r}+\frac{\cos \varphi}{r} \frac{\partial S}{\partial \varphi}\right)\right] \\
(\vec{V} \cdot \nabla) T=\nabla^{2} T \\
(\vec{V} \cdot \nabla) S=L e^{-1} \nabla^{2} S
\end{gathered}
$$

Where $T$ and $S$ are the dimensionless temperature and solute concentration, respectively. $\Psi$ is the stream function defined by: $u=\frac{1}{r} \frac{\partial \Psi}{\partial \varphi}$ and $v=-\frac{\partial \Psi}{\partial r}$, where $u$ and $v$ are the radial and tangential dimensionless velocity components.

In the above equations, the three dimensionless parameters are the Darcy-Rayleigh number, $R a=\frac{g \beta_{T} \Delta T K r_{i}}{v \alpha}$, the buoyancy ratio, $N=\frac{\beta_{S} \Delta S}{\beta_{T} \Delta T}$ and the Lewis number, $L e=\frac{\alpha}{D}$. Where $g$ is the gravitational acceleration, the quantities $\beta_{T}$ and $\beta_{S}$ are thermal and solutal fluid expansion coefficients, $K$ is the permeability of the porous medium, $v$ is the kinematic viscosity, $\alpha$ is the thermal fluid and porous medium and $D$ is the solutal diffusivité.

The dimensionless boundary conditions applied on the active walls are defined as follows:

$$
\begin{aligned}
& r=1, \forall \varphi: T=1, S=1 \text { and } \frac{\partial \Psi}{\partial \varphi}=0 \\
& r=R, \forall \varphi: T=0, S=0 \text { and } \frac{\partial \Psi}{\partial \varphi}=0
\end{aligned}
$$

Where $R$ is the radius ratio defined by $R=\frac{r_{o}}{r_{i}}$.

Taking into account the symmetry of the problem with respect to the vertical plan passing through the axis of cylinders, two others boundary conditions can be added:

$$
\varphi=0 \text { or } \pi, \forall r: \frac{\partial T}{\partial \varphi}=0, \frac{\partial S}{\partial \varphi}=0 \text { and } \frac{\partial \Psi}{\partial r}=0
$$

The average Nusselt and Sherwood numbers are calculated respectively by the expressions: 


$$
\left\{\begin{array}{c}
\overline{N u}=-\left.\frac{1}{\pi} \ln R \int_{0}^{\pi} \frac{\partial T}{\partial r}\right|_{r=1} \\
\overline{S h}=-\left.\frac{1}{\pi} \ln R \int_{0}^{\pi} \frac{\partial S}{\partial r}\right|_{r=1}
\end{array}\right.
$$

The mathematic system Eqs. (1-7) is discretized using Centered Finite Difference method and solved with the Alternating Direction Implicit (ADI) scheme. Three initial conditions types, IC, are employed to initialize the stream function, temperature and solute concentration fields, when starting out the numerical simulation for each given value of Rayleigh number. $I C(I)$ : the temperature and solutal concentration fields corresponding to the pure conduction regime, $I C(I I)$ : the temperature and solutal concentration field can generate the bicellular flow and $I C(I I I)$ : the temperature and solutal concentration field can generate the tricellular flow. Moreover, the velocity profile is initialized with zero for each initial conditions type.

The validation of the numerical accuracy code is performed through a comparison with other investigations available in the literature for the horizontal porous annulus in purely thermal case ( $L e=1$ and $N=0$ ) for the cavity of radius ratio $R$ $=2$. Table 1 shows that a great agreement was found with the literature.

Table 1: Comparison between obtained average Nusselt number values and those published in the literature for $L e=1, N=0, R=2$.

\begin{tabular}{|c|c|c|c|c|c|c|}
\hline \multirow{2}{*}{$R a$} & \multicolumn{3}{|c|}{ Present study } & \multicolumn{3}{c|}{ Others investigation } \\
\cline { 2 - 7 } & $I C(I)$ & $I C(I I)$ & $I C(I I I)$ & $I C(I)$ & $I C(I I)$ & $I C(I I I)$ \\
\hline 150 & 2.3114 & 2.3114 & 2.5463 & $2.295^{[2]}$ & $2.516^{[2]}$ & $2.601^{[2]}$ \\
\hline 200 & 2.6884 & 2.6884 & 2.9257 & $2.6256^{[1]}$ & $2.68^{[8]}$ & $2.657^{[2]}$ \\
\hline 300 & 3.3170 & 3.3170 & 3.5203 & $3.287^{[8]}$ & $(3.48-3.56)^{[8]}$ & $(3.48-3.56)^{[8]}$ \\
\hline
\end{tabular}

\section{Results and Discussion}

\subsection{Flow Pattern}

The initial conditions modes, namely $I C(I), I C(I I)$ and $I C(I I I)$, have no effect on the flow pattern for Rayleigh number values below the critical value $\left(R a \leq R a_{c}\right)$, where the unicellular flow is identical and characterized by a counterclockwise cell occupying the entire annular space. Figure 2 illustrates the streamlines, isotherms and isoconcentration lines within the annular space using the initial condition $I C(I)$ type. Increasing Rayleigh number to the value $R a=47.4$ shows that the unicellular flow develops to unicellular bi-swirling flow, as illustrated in the subfigure 2.a. This unicellular bi-swirling flow occurs over the range $47.4 \leq R a \leq 53.3$. At the critical value $R a_{c}=53.4$, the creation of a new clockwise cell between the two co-rotating cells, see subfigure 2.b, gives rise to the first bifurcation from the unicellular bi-swirling flow to tricellular flow, which persists over the whole range $53.4 \leq R a \leq 70.6$. When $R a$ reaches the second critical value $R a_{c}=$ 70.7, the upper counterclockwise cell is crashed into by clockwise cell below, and the pinching of the primary cell undergoes a new bifurcation from the tricellular flow to bicellular swirling flow, as illustrated in subfigure 2.c. However, this flow pattern is observed for a small range of Rayleigh number $70.7 \leq R a \leq 71.5$. The system under investigation transits from bicellular swirling flow to tetracellular flow at $R a_{c}=71.6$, see subfigure 2.d, through the creating of a clockwise cell between the two counterclockwise cells characterizing the bicellular swirling flow. The tetracellular flow is obtained throughout the whole the range $71.6 \leq R a \leq 365.5$. The same mechanism of bifurcation from tricellular flow to bicellular swirling flow gives rises to a reverse transition from tetracellular flow to bicellular flow at $R a_{c}=365.6$, see subfigure 2.e. This second bicellular flow persists over the entire range $365.6 \leq R a \leq 380$.

Computation with $I C(I I)$ initial conditions type shows that the onset and development of multicellular flows process discussed for the $I C(I)$ type occurs with the same scenario until $R a \leq 53.3$. However, for $I C(I I)$ type, only the bifurcation from unicellular bi-swirling to tricellular flow occurs at $R a_{c}=53.4$. This result is the major interest of this initial condition 
type allowing the prediction of a precise steady-state solution, i.e. tricellular flow, whatever the Rayleigh number value for $R a \geq 53.4$.

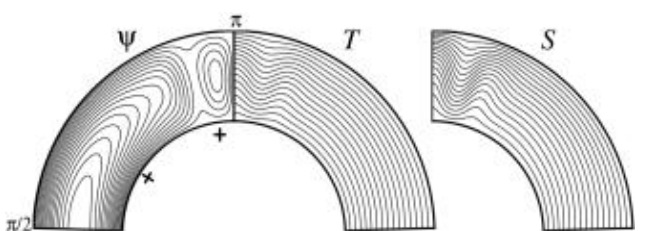

$\Psi_{\max }^{+}=3.7033$

$\overline{N u}=1.0176, \overline{S h}=1.0667$

(a) $R a=50$

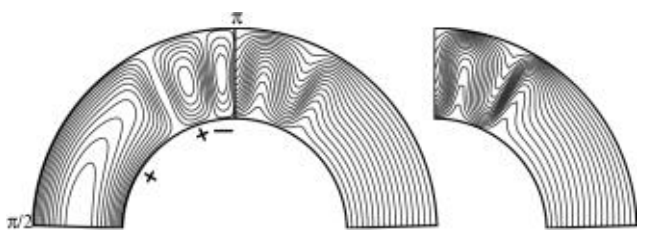

$\Psi_{\max }^{+}=5.2159, \underline{\Psi_{\max }^{-}}=1.8500$

$\overline{N u}=1.0892, \overline{S h}=1.2719$

(c) $R a=71$

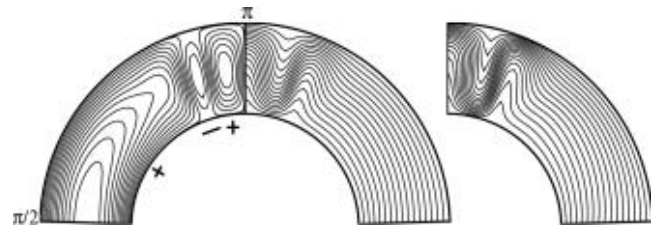

$\Psi_{\max }^{+}=5.1447, \Psi_{\max }^{-}=1.0925$

$\overline{N u}=1.0777, \overline{S h}=1.2391$

(b) $R a=70$

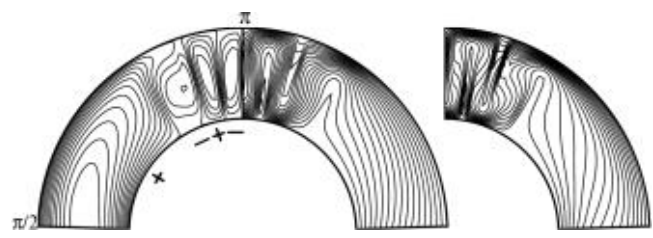

$\Psi_{\max }^{+}=18.7758, \Psi_{\max }^{-}=6.1112$

$\overline{N u}=1.6855, \overline{S h}=2.4691$

(d) $R a=300$

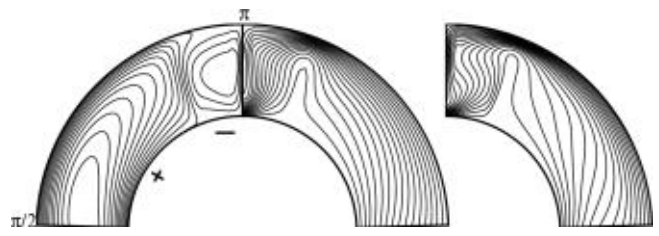

$$
\begin{gathered}
\Psi_{\max }^{+}=22.2263, \Psi_{\max }^{-}=7.1546 \\
\overline{N u}=1.6078, \overline{S h}=2.4132 \\
(e) R a=370
\end{gathered}
$$

Fig. 2: Flow patterns using $I C(I)$ type as initial conditions for $R=1.2, L e=2$ and $N=2$.

Figure 3 shows the obtained results for initial condition $I C(I I I)$ type. Two important remarks are observed for this mode. Firstly, a direct transition is reported from unicellular to bicellular flow, see subfigure 3.a, unlike the two previous initial conditions types, where the unicellular flow transits to the unicellular bi-swirling flow. Also, for the $I C(I I I)$ type, the bifurcation occurs at a low critical value, $R a_{c}=46.5$, as compared with the two other initial conditions types, where $R a_{c}=47.3$. When the Rayleigh number is increased beyond the value $R a=49.7$, the pinching of the main cell gives rise to the bicellular swirling flow, see subfigure 3.b. This flow persists until $R a=54.3$, where a small increases in Rayleigh number to $R a_{c}=54.4$, leads to a transition from bicellular swirling flow to tricellular flow, see subfigure 3.c, across the entire range $54.4 \leq R a \leq 98.3$. Another bifurcation is detected at $R a_{c}=98.4$, where the tricellular flow transits to a first tetracellular flow at $R a_{c}=98.4$. This latter flow structure persists in the whole range $98.4 \leq R a \leq 103.1$, as illustrated in subfigure 3.d. Furthermore, the system under consideration undergoes a reverse transition to bicellular flow (seen Figure 3.e) occuring over the range $R a_{c}=103.2 \leq R a \leq 279.9$. Soon after, at $R a_{c}=$ 280, the system shifts again to a second tetracellular flow, as shown in subfigure 3.f. 


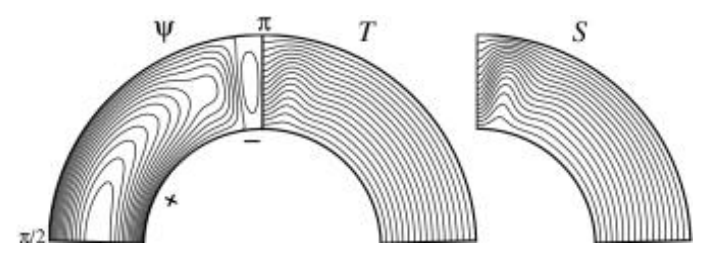

$\Psi_{\text {max }}^{+}=3.6304, \Psi_{\underline{\max }}^{-}=0.5106$

$\overline{N u}=1.0165, \overline{S h}=1.0630$

(a) $R a=49$

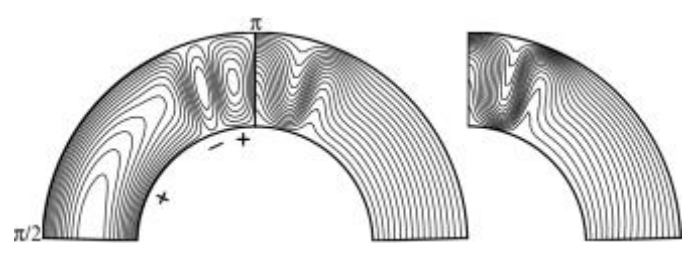

$\Psi_{\max }^{+}=5.8525, \Psi_{\text {max }}^{-}=1.5081$

$\overline{N u}=1.1078, \overline{S h}=1.3118$

(c) $R a=80$

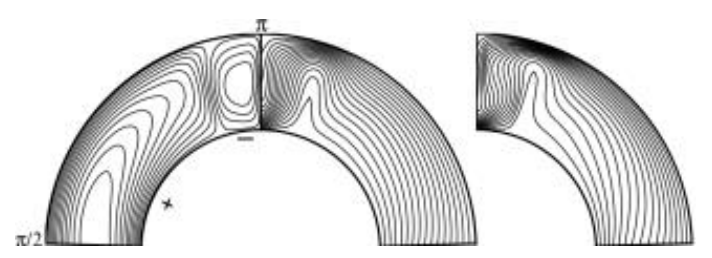

$\Psi_{\max }^{+}=13.5570, \Psi_{\max }^{-}=5.0175$

$\overline{N u}=1.3015, \overline{S h}=1.7767$

(e) $R a=200$

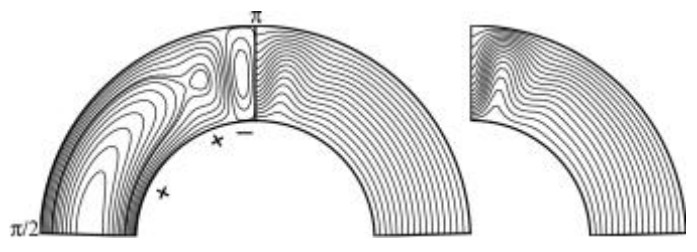

$\Psi_{\text {max }}^{+}=3.9941, \Psi_{\text {max }}^{-}=0.9596$

$\overline{N u}=1.0258, \overline{S h}=1.0935$

(b) $R a=54$

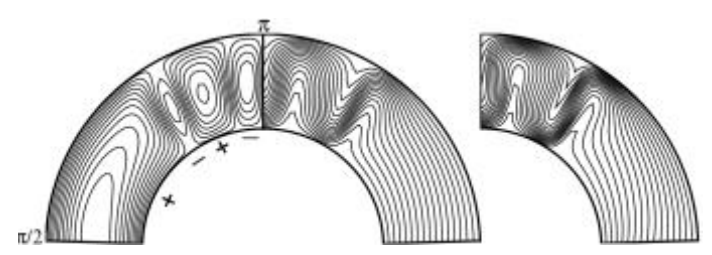

$\Psi_{\max }^{+}=7.234, \Psi_{\max }^{-}=2.9058$

$\overline{N u}=1.2124, \overline{S h}=1.5490$

(d) $R a=100$

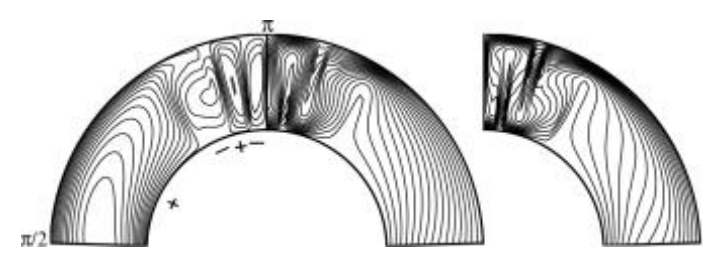

$$
\begin{gathered}
\Psi_{\max }^{+}=22.4798, \Psi_{\max }^{-}=6.8329 \\
\overline{N u}=1.8274, \overline{S h}=2.7327 \\
\text { (f) } R a=380
\end{gathered}
$$

Fig. 3: Flow patterns for $I C(I I I)$ type, $R=1.2, L e=2$ and $N=2$.

\subsection{Flow Intensity}

Figure 4 illustrates the variation of the maximum stream function, $\psi_{\text {max }}^{+}$, corresponding to the counterclockwise cells and $\psi_{\max }^{-}$, corresponding to the clockwise cells with respect to the Rayleigh number value. The above descriptions of the transition between the different flow structures explain clearly the variation of the stream function behavior with increasing Rayleigh number. Figure 4 shows a linear increase in $\psi_{\max }^{+}$with increasing $R a$, which is the natural effect of thermosolutal convection increasingly important. In this context, a perfect matching of $\psi_{\max }^{+}$was observed between the three initial conditions types. So, the maximum stream function of the counterclockwise cells, $\psi_{\max }^{+}$, is not influenced by the nature of the initial conditions whatever the $R a$ values.

The challenge in studying initial conditions effect on thermosolutal natural convection in the confined porous media comes mainly from the inherent variety of the flow structures and difficulty of their further development. The transition from the unicellular bi-swirling flow $\left(\psi_{\max }^{-}=0\right)$ to the tricellular flow at the critical value $R a_{c}=53.4$ for $I C(I)$ type is associated with an increase in $\psi_{\max }^{-}$. On the other hand, the reverse transition from tricellular flow to bicellular swirling flow at $R a_{c}=70.7$, is associated with the increase of the clockwise cell size, which causes an enhancement of the $\psi_{\max }^{-}$ $(38.58 \%)$. Also, the maximal stream function of the clockwise cells, $\psi_{\max }^{-}$, is improved again by $5.79 \%$ through the bifurcation from tetracellular flow to bicellular flow at $R a_{c}=365.6$. 


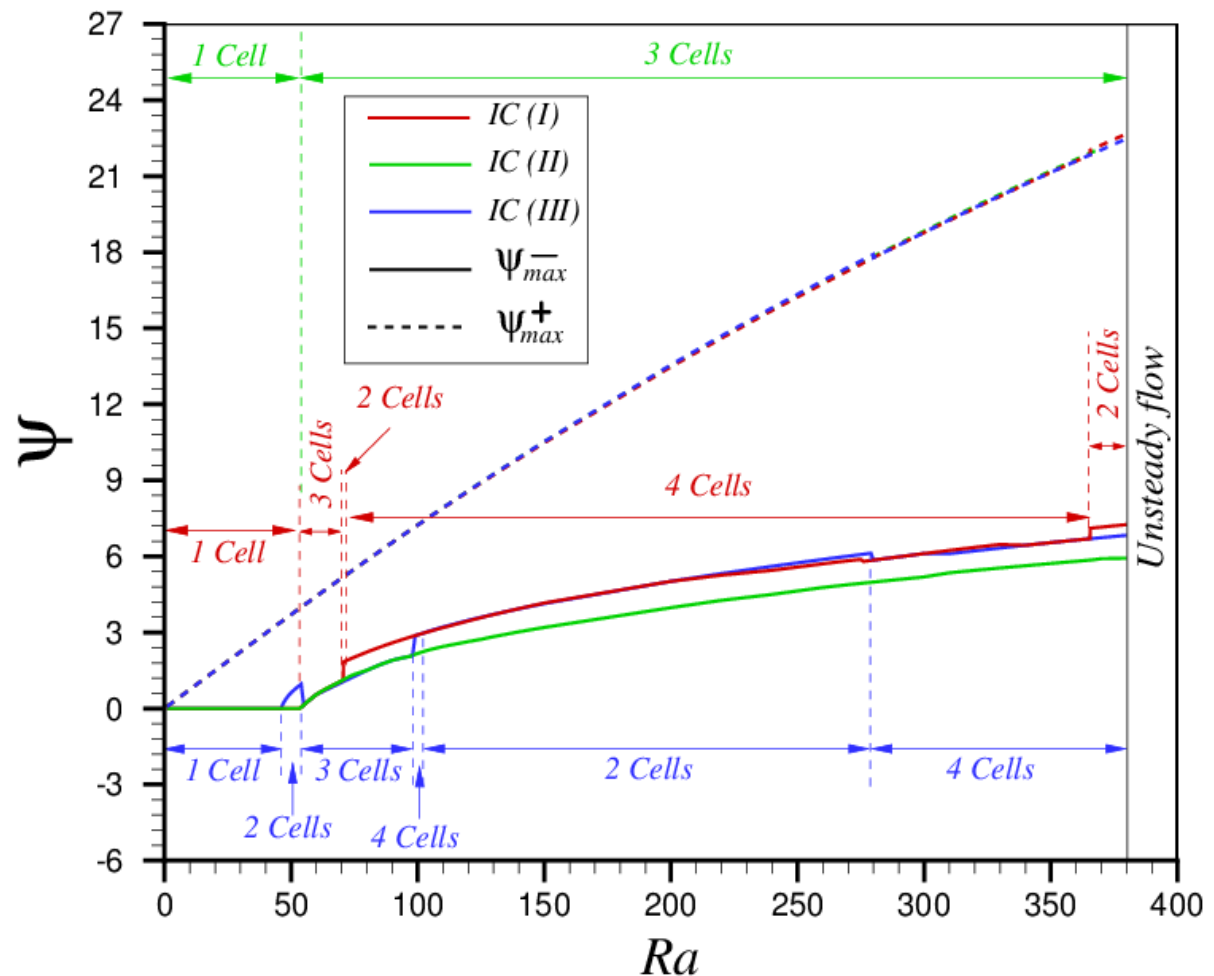

Fig. 4: Evolution of $\psi_{\max }^{+}$and $\psi_{\max }^{-}$using the three initial conditions types with respect to $R a$, for $R=1.2, L e=2$ and $N=2$.

However, the introduction of $I C(I I)$ and $I C(I I I)$ modes implies different behaviors of $\psi_{\max }^{-}$compared to those obtained for $I C(I)$ type. As discussed previously, the $I C(I)$ and $I C(I I)$ have the same flow pattern for all the Rayleigh number range $R a \leq 70.6$, i.e., unicellular, unicellular bi-swirling, and tricellular flow. This gives the same variation of $\psi_{\max }^{-}$for both modes. However, the tricellular flow obtained with $I C(I I)$ mode persists over the range $R a \geq 70.7$, which results in a progressive increase of $\psi_{\max }^{-}$with respect to $R a$, as shown in Figure 4.

A complex behavior of $\psi_{\max }^{-}$is obtained using $I C(I I I)$ type. First, the transition from unicellular flow to bicellular flow at the critical value $R a_{c}=46.5$, enhances $\psi_{\max }^{-}$, as illustrated in Figure 4. The bifurcation to tricellular flow at the value $R a_{c}=54.4$ shows a great decrease in $\psi_{\max }^{-}(-91,45 \%)$, due to reduction in clockwise cell size. Furthermore, an identical $\psi_{\max }^{-}$variation is observed for both initial conditions $I C(I I)$ and $I C(I I I)$ modes over the range $54.4 \leq R a \leq 98.3$ where the flow pattern is tricellular. Moreover, the transition of the flow pattern based on IC(III) type from tricellular flow to tetracelular flow at the critical value $R a_{c}=98.4$, allows an almost identical $\psi_{\max }^{-}$variation with $I C(I)$ mode, over the entire range $R a_{c}=98.4 \leq R a \leq 365.5$, even though calculations initialized with both initial conditions have different flow structures. The maximum deviation obtained between both initial conditions in this considered Rayleigh number range is $4.91 \%$. However, the bifurcation obtained with $I C(I)$ type to the bicellular flow at $R a_{c}=356.6$ involves a remarkable difference between the three initial conditions: $17.64 \%$ between $I C(I)$ and $I C(I I)$, and $5.78 \%$ between $I C(I)$ and $I C(I I I)$ at the value $R a=356.6$.

\subsection{Heat and Mass Transfer Rates}

Heat and mass transfer rates, i.e., average Nusselt number, $\overline{N u}$, and Sherwood number, $\overline{S h}$, are evaluated as a functions of Rayleigh number using the three initial conditions types. The obtained results are displayed in Figure 5 for $\overline{N u}$ (here $\overline{S h}$ has the same behaviors). As it is shown, the overall heat transfer rates are identical for the three initial conditions types when the $R a \leq 46.4$, which corresponds to the unicellular flow. Also, $\overline{N u}$ is similar for $I C(I)$ and $I C(I I)$ when the structure is unicellular bi-swirling flow, $47.4 \leq R a \leq 53.3$, and tricellular flow, 53.4 $\leq R a \leq 70.6$. Meanwhile, the transition of the flow patterns using $I C(I I I)$ type from unicellular flow to bicellular flow, $R a_{c}=46.5 \leq R a \leq 49.6$, and bicellular swirling flow, $49.7 \leq R a \leq 54.3$, does not involves a feature of interest energy and mass transfers compared to the other initial 
conditions mode $(0.38 \%)$. The bifurcations observed with the $I C(I)$ type from tricellular flow to bicellular swirling flow at $R a_{c}=70.7$ and to tetracellular flow at $R a_{c}=71.6$ allows an enhancement of the average heat transfer rate, which represents a significant energy interest for the $I C(I)$ types over the range $70.7 \leq R a \leq 365.5$. However, the reverse transition from the tetracellular flow to bicellular flow at the critical value $R a_{c}=365.6$ involves a remarkable reduction of the heat transfer rate, $-11.09 \%$. In comparison, the linear increase of $\overline{N u}$ for the $I C(I I)$ mode shows that the only possible flow for this mode beyond the critical value $R a_{c}=53.4$ is the tricellular flow. In addition, as a direct result of increasing $R a$, the thermosolutal convection becomes more important.

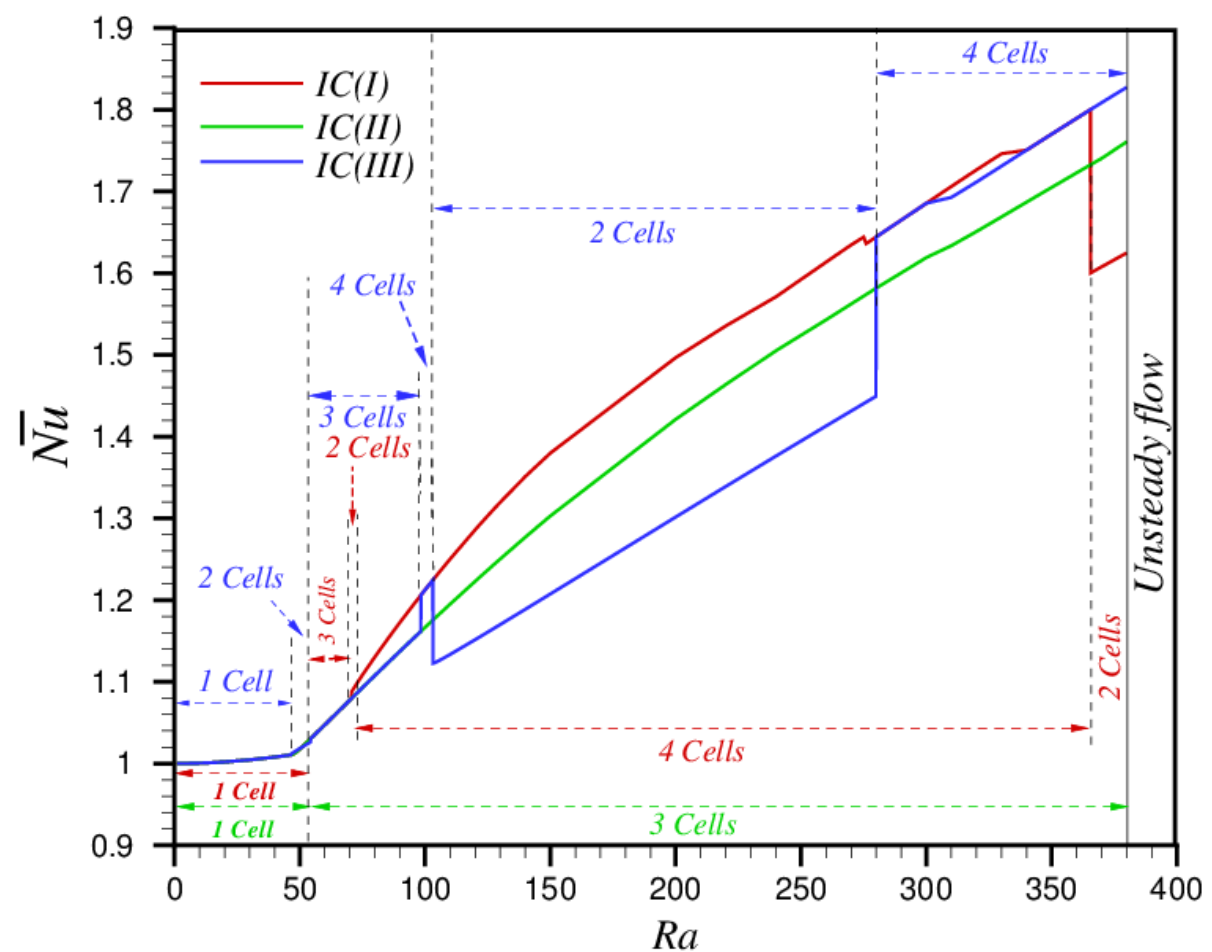

Fig. 5: Evolution of $\overline{N u}$ as function of $R a$.

For the $I C(I I I)$ mode, the transition from the tricellular flow to tetracellular flow at $R a_{c}=98.4$ leads to a sudden increase in $\overline{N u}$ by $3.69 \%$. On the entire range $98.4 \leq R a \leq 103.1$, the evolution profiles of $\overline{N u}$ values with respect to $R a$ using the $I C(I)$ and $I C(I I I)$ types are perfectly identical. Reducing the number of the convective cells when the system under consideration bifurcates at $R a_{c}=103.2$ using $I C(I I I)$ mode from tetracellular flow to the bicellular swirling flow involves a falling of thermal transfer rate to achieve $-8.31 \%(-14.77 \%$ for $\overline{S h})$ at this critical value of $R a$. Upon the whole range $103.2 \leq R a \leq 279.9$, the $I C(I I I)$ mode represents the lowest transfer rate compared to the $I C(I)$ and $I C(I I)$ modes of $13.03 \%$, and $8.41 \%$, respectively. Although, improvements of the transfer rate are recognized through the return from the bicellular flow to tetracellular flow at $R a_{c}=280,13.45 \%$. In this situation, the two modes $I C(I)$ and $I C(I I I)$ are in perfect agreement. Energy interest of the $I C(I I I)$ mode is observed for $R a \geq 365.6$, where only $I C(I I I)$ mode provides a maximum number of convective cells (tetracellular flow), leading to the highest thermal and solutal transfer rates compared to the other $I C$ 's modes, $11.11 \%$ and $3.75 \%$, at $R a=365.6$, respectively for $I C(I)$.

\section{Conclusion}

The present investigation involves the numerical simulation of initial conditions effect on flow patterns and heat and mass transfer rates in a two-dimensional horizontal annular $R=1.2$. Centered Finite Differences method has been used for 
the discretization of the governing equations and solved with the ADI scheme. For the calculations initialized with $I C(I)$ and $I C(I I I)$ types, increasing the $R a$ value involves various flow structures, where the critical Rayleigh number value for each transition is determined with accuracy. While the $I C(I I)$ type is relatively stable compared with others. Furthermore, the heat and mass transfer behaviors are heavily depend to the initial conditions used and the Rayleigh number value. For the $I C(I)$ type, energy and mass transfers interest is observed over the range $70.7 \leq R a \leq 365.5$, where the average Nusselt and Sherwood numbers reach their maximum value compared to other initial conditions. However, the IC(III) type allows a maximum transfer rate for $R a \geq 365.6$.

\section{References}

[1] J. P. Caltagirone, "Thermoconvective instabilities in a porous medium bounded by two concentric horizontal cylinders," Journal of fluid Mechanics, vol. 76, no. 2, pp. 337-362, 1976.

[2] Y. F. Rao, K. Fukuda and S. Hasegawa, "Steady and transient analyses of natural convection in a Horizontal Porous Annulus With the Galerkin Method," Journal of Heat Transfer, vol. 109, pp. 919-927, 1987.

[3] J. P. B. Mota and E. Saatdjian, "Natural convection in porous horizontal cylindrical annulus," ASME J. of Heat Transfer, vol. 116, pp. 621-626, 1994

[4] J. P. B. Mota and E. Saatdjian, "Natural convection in porous cylindrical annuli," International Journal of Numerical Methods for Heat Fluid Flow, vol. 5, no. 1, pp. 3-12, 1995.

[5] M. C. Charrier-Mojtabi, "Numerical simulation of two- and three dimensional free convection flows in a horizontal porous annulus using a pressure and temperature formulation," Int. J. Heat Mass Transfer, vol. 40, no. 7, pp. 1521$1533,1997$.

[6] N. D. Seurtu, A. Postelnicu and I. Pop, "Free convection between two horizontal concentric cylinders filled with a porous medium a perturbed solution," Acta Mechanica, vol. 151, pp. 115-125, 2001.

[7] J. Belabid, and A. Cheddadi, "Natural convection in an annular porous medium: effect of brinkman correction on the bifurcation point, Applied Mechanics and Materials, vols. 789-790, pp. 403-406, 2015.

[8] M. C. Charrier-Mojtabi, A. Mojtabi, M. Azaiez and G. Labrosse, "Numerical and experimental study of multicellular free convection flows in an annular porous layer," International journal of Heat and Mass Transfer, vol. 34, no. 12, pp. 3061-3074, 1991. 